\title{
Signal Processing for Nondifferentiable Data Defined on Cantor Sets: A Local Fractional Fourier Series Approach
}

\author{
Zhi-Yong Chen, ${ }^{1}$ Carlo Cattani, ${ }^{2}$ and Wei-Ping Zhong $^{3}$ \\ ${ }^{1}$ School of Electronic Science and Engineering, National University of Defense Technology, Changsha 410073, China \\ ${ }^{2}$ Department of Mathematics, University of Salerno, Via Giovanni Paolo II, Fisciano, 84084 Salerno, Italy \\ ${ }^{3}$ School of Mechanics \& Civil Engineering, China University of Mining \& Technology, Xuzhou 221116, China
}

Correspondence should be addressed to Carlo Cattani; ccattani@unisa.it

Received 3 May 2014; Revised 13 May 2014; Accepted 13 May 2014; Published 10 June 2014

Academic Editor: Xiao-Jun Yang

Copyright (C) 2014 Zhi-Yong Chen et al. This is an open access article distributed under the Creative Commons Attribution License, which permits unrestricted use, distribution, and reproduction in any medium, provided the original work is properly cited.

From the signal processing point of view, the nondifferentiable data defined on the Cantor sets are investigated in this paper. The local fractional Fourier series is used to process the signals, which are the local fractional continuous functions. Our results can be observed as significant extensions of the previously known results for the Fourier series in the framework of the local fractional calculus. Some examples are given to illustrate the efficiency and implementation of the present method.

\section{Introduction}

Fractional derivatives $[1,2]$, like the Caputo derivative, the Riemann-Liouville derivative, and the Grünwald-Letnikov derivative, were applied to model some anomalous phenomena, such as the anomalous diffusion $[3,4]$, Brownian motion [5], relaxation in dielectrics [6], transport of particles [7], and reaction kinetics [8]. From the signal processing point of view, the fractional-order signal processing is anomalous behavior of nature from practice activity. In literature [9-16], many researchers employed the fractional calculus theory to handle signals, which are continuous characteristics (having a similar behavior). Some applications of the fractional-order signal processing to electrochemical noises were presented [15]. In [16], Tao and coauthors suggested the signal processing by using the fractional Fourier transform. The short time fractional Fourier transform was used to handle the robotic manipulators with vibrations in [17]. The fractional wavelet transform for processing the composite signals of the active compounds was considered in [18].

There is also a class of signals of the random sequences, which have a fractal behavior, and some methods were suggested in [19-21]. Some signals are defined on the Cantor sets, such as the Cantor function and Cantor-like functions, which are nondifferentiable data.
Figure 1 shows an example of a signal for a Cantor function object while in Figures 2 and 3 there are some examples of signals on the Cantor-like functions defined on the Cantor sets.

With these signals being defined on Cantor sets, classical methods of signal analysis are not efficient to deal with them. To overcome these drawbacks, suitable methods for the signals on the Cantor sets are developed, such as the local fractional Fourier series [22-27], wavelet transform [28], and its discrete version [29].

In view of the special characteristics of the local fractional Fourier series [22, 23], as alternative method for Fourier series based upon the local fractional calculus, the aim of this paper is to investigate the signal processing of nondifferentiable data defined on the Cantor sets, which is a special case of local fractional continuous function [30]. The paper has been organized as follows. Section 2 gives the fundamental concepts of local fractional Fourier series. In Section 3, the nondifferentiable data defined on the Cantor sets are processed. Conclusions are given in Section 4.

\section{Mathematical Tools}

In this section, we present the fundamental concepts for the local fractional Fourier series and some results for the local 


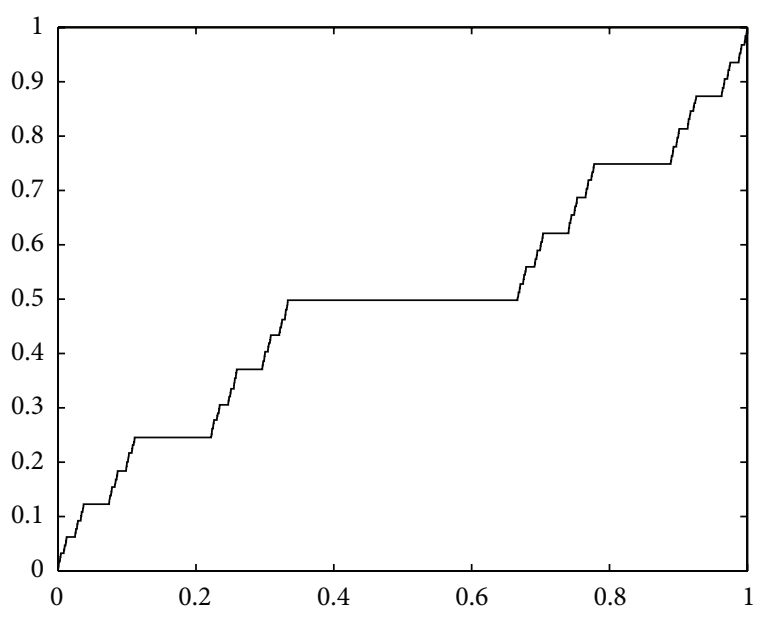

Figure 1: The Cantor function.

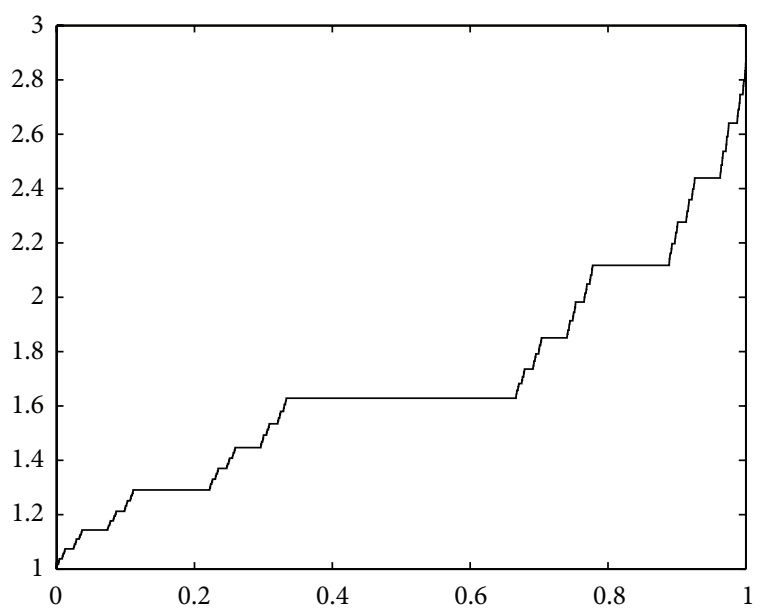

FIgure 2: The Cantor-like function defined on the Cantor sets.

fractional integral operator [22-27], which are used in this paper.

If, for $x \in(a, b)$, a function $f(x)$ fulfills the condition

$$
\left|f(x)-f\left(x_{0}\right)\right|<\varepsilon^{\alpha},
$$

with $\left|x-x_{0}\right|<\delta$, for $\varepsilon, \delta>0$ and $\varepsilon, \delta \in R$, then $f(x) \epsilon$ $C_{\alpha}(a, b)$; namely, it is the so-called local fractional continuous on the interval $(a, b)$. If the fractal dimension $\alpha$ is equal to 1 , this definition reduces to the classical one.

Let $f(x) \in C_{\alpha}(-\infty, \infty)$. Local fractional trigonometric Fourier series of $f(x)$ is given by [22-27]

$$
\begin{aligned}
f(x)= & a_{0}+\sum_{k=1}^{\infty} a_{k} \sin _{\alpha}\left(k^{\alpha} \omega_{0}^{\alpha} x^{\alpha}\right) \\
& +\sum_{k=1}^{\infty} b_{k} \cos _{\alpha}\left(k^{\alpha} \omega_{0}^{\alpha} x^{\alpha}\right) .
\end{aligned}
$$

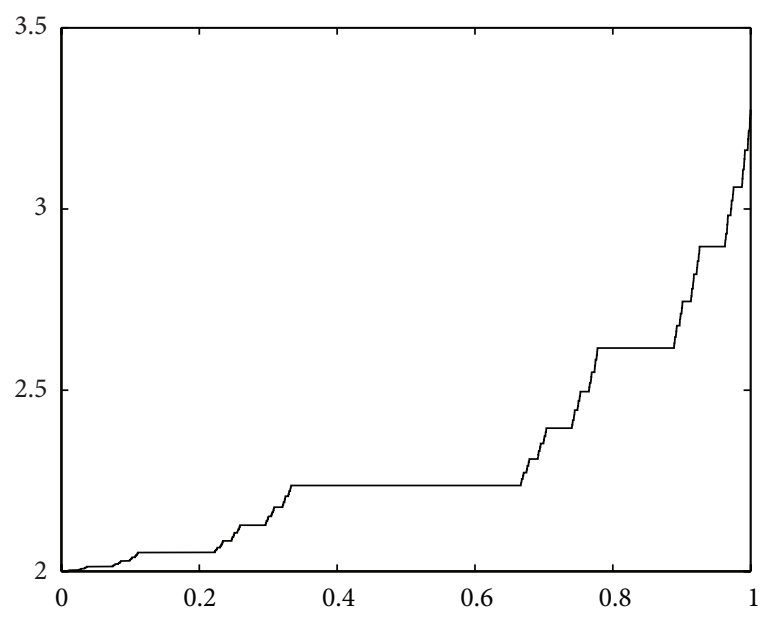

FIgURE 3: Another Cantor-like function defined on the same Cantor sets.

The local fractional Fourier coefficients read as follows [2227]:

$$
\begin{aligned}
& a_{0}=\frac{\Gamma(1+\alpha)}{T^{\alpha}}{ }_{0} I_{T}^{(\alpha)} f(x), \\
& a_{k}=\frac{2^{\alpha} \Gamma(1+\alpha)}{T^{\alpha}}{ }_{0} I_{T}^{(\alpha)} f(x) \sin _{\alpha}\left(k^{\alpha} \omega_{0}^{\alpha} x^{\alpha}\right), \\
& b_{k}=\frac{2^{\alpha} \Gamma(1+\alpha)}{T^{\alpha}}{ }_{0} I_{T}^{(\alpha)} f(x) \cos _{\alpha}\left(k^{\alpha} \omega_{0}^{\alpha} x^{\alpha}\right),
\end{aligned}
$$

where the local fractional integral of $f(x)$ of order $\alpha$ in the interval $[a, b]$ is defined as [22-30]

$$
\begin{aligned}
a_{b}^{(\alpha)} f(x) & =\frac{1}{\Gamma(1+\alpha)} \int_{a}^{b} f(t)(d t)^{\alpha} \\
& =\frac{1}{\Gamma(1+\alpha)} \lim _{\Delta t \rightarrow 0} \sum_{j=0}^{j=N-1} f\left(t_{j}\right)\left(\Delta t_{j}\right)^{\alpha},
\end{aligned}
$$

where the partition of the interval $[a, b]$ is denoted as $\Delta t_{j}=$ $t_{j+1}-t_{j}, \Delta t=\max \left\{\Delta t_{0}, \Delta t_{1}, \ldots, \Delta t_{j}, \ldots\right\}$, and $j=0, \ldots, N-$ $1, t_{0}=a$, and $t_{N}=b$. For more details on the local fractional Fourier series, see [22-27].

The Lebesgue-Cantor staircase function is defined by [30]

$$
H_{\alpha}(F \cap(0, x))=\Gamma(1+\alpha)_{0} I_{x}^{(\alpha)} 1,
$$

where $F$ is a Cantor set, $H_{\alpha}(\cdot)$ is the $\alpha$ dimensional Hausdorff measure, $I_{x}^{(\alpha)}(\cdot)$ is the local fractional integral operator [24$30]$, and $\Gamma(\cdot)$ is the Gamma function.

Following (7), we obtain $[24,30]$

$$
H_{\alpha}(F \cap(0, x))=x^{\alpha},
$$

which is the Lebesgue-Cantor staircase function. 
Advances in Mathematical Physics

3

Some useful formulas, which are used in this paper, are presented as follows [30]:

$$
\begin{aligned}
& { }_{a} I_{b}^{(\alpha)}[f(x) \pm g(x)]={ }_{a} I_{b}^{(\alpha)} f(x) \pm{ }_{a} I_{b}^{(\alpha)} g(x), \\
& { }_{a} I_{b}^{(\alpha)}[C f(x)]=C_{a} I_{b}^{(\alpha)} f(x), \\
& { }_{a} I_{b}^{(\alpha)} f(x)=g(b)-g(a), \\
& { }_{a} I_{x}^{(\alpha)} f(x) g^{(\alpha)}(x) \\
& =\left.[f(x) g(x)]\right|_{a} ^{x}-{ }_{a} I_{x}^{(\alpha)} f^{(\alpha)}(x) g(x), \\
& { }_{0} I_{x}^{(\alpha)} E_{\alpha}\left(x^{\alpha}\right)=E_{\alpha}\left(x^{\alpha}\right)-1 \text {, } \\
& { }_{0} I_{x}^{(\alpha)} \sin _{\alpha}\left(a^{\alpha} x^{\alpha}\right)=\frac{1}{a^{\alpha}}\left[\cos _{\alpha}\left(a^{\alpha} x^{\alpha}\right)-1\right], \\
& { }_{0} I_{x}^{(\alpha)} \cos _{\alpha}\left(a^{\alpha} x^{\alpha}\right)=\frac{1}{a^{\alpha}} \sin _{\alpha}\left(a^{\alpha} x^{\alpha}\right), \\
& { }_{0} I_{x}^{(\alpha)} \frac{x^{\alpha}}{\Gamma(1+\alpha)} \sin _{\alpha}\left(a^{\alpha} x^{\alpha}\right) \\
& =-\frac{1}{a^{\alpha}}\left[\frac{x^{\alpha}}{\Gamma(1+\alpha)} \cos _{\alpha}\left(a^{\alpha} x^{\alpha}\right)-\frac{1}{a^{\alpha}} \sin _{\alpha}\left(a^{\alpha} x^{\alpha}\right)\right], \\
& { }_{0} I_{x}^{(\alpha)} \frac{x^{\alpha}}{\Gamma(1+\alpha)} \cos _{\alpha}\left(a^{\alpha} x^{\alpha}\right) \\
& =\frac{1}{a^{\alpha}}\left\{\frac{x^{\alpha}}{\Gamma(1+\alpha)} \sin _{\alpha}\left(a^{\alpha} x^{\alpha}\right)\right. \\
& \left.-\frac{1}{a^{\alpha}}\left[\cos _{\alpha}\left(a^{\alpha} x^{\alpha}\right)-1\right]\right\},
\end{aligned}
$$$$
{ }_{0} I_{x}^{(\alpha)} \frac{x^{2 \alpha}}{\Gamma(1+2 \alpha)} \sin _{\alpha}\left(a^{\alpha} x^{\alpha}\right)
$$$$
=-\frac{1}{a^{\alpha}} \frac{x^{2 \alpha}}{\Gamma(1+2 \alpha)} \cos _{\alpha}\left(a^{\alpha} x^{\alpha}\right)
$$$$
+\frac{1}{a^{2 \alpha}}\left\{\frac{x^{\alpha}}{\Gamma(1+\alpha)} \sin _{\alpha}\left(a^{\alpha} x^{\alpha}\right)\right.
$$$$
\left.-\frac{1}{a^{\alpha}}\left[\cos _{\alpha}\left(a^{\alpha} x^{\alpha}\right)-1\right]\right\} \text {, }
$$

$$
\begin{aligned}
{ }_{0} I_{x}^{(\alpha)} & \frac{x^{2 \alpha}}{\Gamma(1+2 \alpha)} \cos _{\alpha}\left(a^{\alpha} x^{\alpha}\right) \\
= & \frac{1}{a^{\alpha}} \frac{x^{2 \alpha}}{\Gamma(1+2 \alpha)} \sin _{\alpha}\left(a^{\alpha} x^{\alpha}\right) \\
& +\frac{1}{a^{2 \alpha}}\left[\frac{x^{\alpha}}{\Gamma(1+\alpha)} \cos _{\alpha}\left(a^{\alpha} x^{\alpha}\right)-\frac{1}{a^{\alpha}} \sin _{\alpha}\left(a^{\alpha} x^{\alpha}\right)\right],
\end{aligned}
$$

$$
\begin{aligned}
& { }_{0} I_{x}^{(\alpha)} \frac{x^{3 \alpha}}{\Gamma(1+2 \alpha)} \sin _{\alpha}\left(a^{\alpha} x^{\alpha}\right) \\
& =-\frac{1}{a^{\alpha}} \frac{x^{3 \alpha}}{\Gamma(1+2 \alpha)} \cos _{\alpha}\left(a^{\alpha} x^{\alpha}\right) \\
& +\frac{1}{a^{2 \alpha}} \frac{x^{2 \alpha}}{\Gamma(1+2 \alpha)} \sin _{\alpha}\left(a^{\alpha} x^{\alpha}\right) \\
& +\frac{1}{a^{3 \alpha}}\left[\frac{x^{\alpha}}{\Gamma(1+\alpha)} \cos _{\alpha}\left(a^{\alpha} x^{\alpha}\right)\right. \\
& \left.-\frac{1}{a^{\alpha}} \sin _{\alpha}\left(a^{\alpha} x^{\alpha}\right)\right], \\
& { }_{0} I_{x}^{(\alpha)} \frac{x^{3 \alpha}}{\Gamma(1+3 \alpha)} \cos _{\alpha}\left(a^{\alpha} x^{\alpha}\right) \\
& =\frac{1}{a^{\alpha}} \frac{x^{3 \alpha}}{\Gamma(1+3 \alpha)} \sin _{\alpha}\left(a^{\alpha} x^{\alpha}\right) \\
& -\frac{1}{a^{2 \alpha}} \frac{x^{2 \alpha}}{\Gamma(1+2 \alpha)} \cos _{\alpha}\left(a^{\alpha} x^{\alpha}\right) \\
& +\frac{1}{a^{3 \alpha}}\left\{\frac{x^{\alpha}}{\Gamma(1+\alpha)} \sin _{\alpha}\left(a^{\alpha} x^{\alpha}\right)\right. \\
& \left.-\frac{1}{a^{\alpha}}\left[\cos _{\alpha}\left(a^{\alpha} x^{\alpha}\right)-1\right]\right\}, \\
& { }_{0} I_{x}^{(\alpha)} \frac{x^{4 \alpha}}{\Gamma(1+4 \alpha)} \sin _{\alpha}\left(a^{\alpha} x^{\alpha}\right) \\
& =-\frac{1}{a^{\alpha}} \frac{x^{4 \alpha}}{\Gamma(1+4 \alpha)} \cos _{\alpha}\left(a^{\alpha} x^{\alpha}\right) \\
& +\frac{1}{a^{2 \alpha}} \frac{x^{3 \alpha}}{\Gamma(1+3 \alpha)} \sin _{\alpha}\left(a^{\alpha} x^{\alpha}\right) \\
& -\frac{1}{a^{3 \alpha}} \frac{x^{2 \alpha}}{\Gamma(1+2 \alpha)} \cos _{\alpha}\left(a^{\alpha} x^{\alpha}\right) \\
& +\frac{1}{a^{4 \alpha}}\left\{\frac{x^{\alpha}}{\Gamma(1+\alpha)} \sin _{\alpha}\left(a^{\alpha} x^{\alpha}\right)\right. \\
& \left.-\frac{1}{a^{\alpha}}\left[\cos _{\alpha}\left(a^{\alpha} x^{\alpha}\right)-1\right]\right\}, \\
& { }_{0} I_{x}^{(\alpha)} \frac{x^{4 \alpha}}{\Gamma(1+4 \alpha)} \cos _{\alpha}\left(a^{\alpha} x^{\alpha}\right) \\
& =\frac{1}{a^{\alpha}} \frac{x^{4 \alpha}}{\Gamma(1+4 \alpha)} \sin _{\alpha}\left(a^{\alpha} x^{\alpha}\right) \\
& -\frac{1}{a^{2 \alpha}} \frac{x^{3 \alpha}}{\Gamma(1+3 \alpha)} \cos _{\alpha}\left(a^{\alpha} x^{\alpha}\right) \\
& +\frac{1}{a^{3 \alpha}} \frac{x^{2 \alpha}}{\Gamma(1+2 \alpha)} \sin _{\alpha}\left(a^{\alpha} x^{\alpha}\right)
\end{aligned}
$$




$$
\begin{array}{r}
+\frac{1}{a^{4 \alpha}}\left[\frac{x^{\alpha}}{\Gamma(1+\alpha)} \cos _{\alpha}\left(a^{\alpha} x^{\alpha}\right)\right. \\
\left.-\frac{1}{a^{\alpha}} \sin _{\alpha}\left(a^{\alpha} x^{\alpha}\right)\right], \\
{ }_{0} I_{x}^{(\alpha)} \frac{x^{k \alpha}}{\Gamma(1+k \alpha)}=\frac{x^{(k+1) \alpha}}{\Gamma[1+(k+1) \alpha]} .
\end{array}
$$

\section{Signal Processing for Data Defined on the Cantor Sets}

This section deals with the nondifferentiable data defined on the Cantor sets. Some examples of nondifferentiable functions defined on the Cantor sets are given and the corresponding local fractional Fourier series are explicitly computed.

Example 1. We present the nondifferentiable data defined on the Cantor sets in the following form:

$$
f(t)=\frac{t^{\alpha}}{\Gamma(1+\alpha)}, \quad(0 \leq t \leq 2 \pi),
$$

where the fractal dimension $\alpha$ is equal to $\ln 2 / \ln 3$.

Using (3), (4), and (5), we have the local fractional Fourier coefficients as follows:

$$
\begin{aligned}
a_{0} & =\frac{\Gamma(1+\alpha)}{(2 \pi)^{\alpha}}{ }_{0} I_{2 \pi}^{(\alpha)} f(x)=\frac{\Gamma(1+\alpha)}{(2 \pi)^{\alpha}}{ }_{0} I_{2 \pi}^{(\alpha)} \frac{t^{\alpha}}{\Gamma(1+\alpha)} \\
& =\frac{\Gamma(1+\alpha)}{\Gamma(1+2 \alpha)}(2 \pi)^{\alpha} \\
a_{k} & =\frac{\Gamma(1+\alpha)}{\pi^{\alpha}}{ }_{0} I_{2 \pi}^{(\alpha)} f(x) \sin _{\alpha}\left(k^{\alpha} x^{\alpha}\right) \\
& =\frac{\Gamma(1+\alpha)}{\pi^{\alpha}}{ }_{0} I_{2 \pi}^{(\alpha)} \frac{t^{\alpha}}{\Gamma(1+\alpha)} \sin _{\alpha}\left(k^{\alpha} x^{\alpha}\right) \\
& =-\left.\left[\frac{1}{k^{\alpha}}\left[\frac{x^{\alpha}}{\Gamma(1+\alpha)} \cos _{\alpha}\left(k^{\alpha} x^{\alpha}\right)-\frac{1}{k^{\alpha}} \sin _{\alpha}\left(k^{\alpha} x^{\alpha}\right)\right]\right]\right|_{0} ^{2 \pi} \\
& =-\left(\frac{2}{k}\right)^{\alpha}, \\
b_{k} & =\frac{\Gamma(1+\alpha)}{\pi^{\alpha}} I_{2 \pi}^{(\alpha)} f(x) \cos _{\alpha}\left(k^{\alpha} x^{\alpha}\right) \\
& \left.=\frac{\Gamma(1+\alpha)}{\pi^{\alpha}}{ }_{0}^{(\alpha)} \frac{1}{k^{\alpha}}\left[t^{\alpha} \cos _{\alpha}\left(k^{\alpha} x^{\alpha}\right)-1\right]\right\}\left.\right|_{0} ^{2 \pi}=0 . \\
& =\left[\frac { 1 } { k ^ { \alpha } } \left\{\frac{x^{\alpha}}{\Gamma(1+\alpha)} \sin _{\alpha}\left(k^{\alpha} x^{\alpha}\right)\right.\right.
\end{aligned}
$$

Hence, from (18)-(19), $f(t)$ is expressed as follows:

$$
f(x)=\frac{\Gamma(1+\alpha)}{\Gamma(1+2 \alpha)}(2 \pi)^{\alpha}-\sum_{k=1}^{\infty}\left(\frac{2}{k}\right)^{\alpha} \sin _{\alpha}\left(k^{\alpha} x^{\alpha}\right) .
$$

Example 2. Let us consider the nondifferentiable data defined on the Cantor sets in the following form:

$$
f(t)=\frac{t^{2 \alpha}}{\Gamma(1+2 \alpha)}, \quad(0 \leq t \leq 2 \pi),
$$

where the fractal dimension $\alpha$ is equal to $\ln 2 / \ln 3$.

According to (3) and (16), we obtain the local fractional Fourier series as follows:

$$
\begin{aligned}
a_{0} & =\frac{\Gamma(1+\alpha)}{(2 \pi)^{\alpha}}{ }_{0} I_{2 \pi}^{(\alpha)} f(x)=\frac{\Gamma(1+\alpha)}{(2 \pi)^{\alpha}}{ }_{0} I_{2 \pi}^{(\alpha)} \frac{t^{2 \alpha}}{\Gamma(1+2 \alpha)} \\
& =\frac{\Gamma(1+\alpha)}{\Gamma(1+3 \alpha)}(2 \pi)^{2 \alpha} .
\end{aligned}
$$

From (4) and (10), we have the following local fractional Fourier series coefficient:

$$
\begin{aligned}
a_{k}= & \frac{\Gamma(1+\alpha)}{\pi^{\alpha}}{ }_{0} I_{2 \pi}^{(\alpha)} f(x) \sin _{\alpha}\left(k^{\alpha} x^{\alpha}\right) \\
= & \frac{\Gamma(1+\alpha)}{\pi^{\alpha}}{ }_{0} I_{2 \pi}^{(\alpha)} \frac{t^{2 \alpha}}{\Gamma(1+2 \alpha)} \sin _{\alpha}\left(k^{\alpha} x^{\alpha}\right) \\
= & \frac{\Gamma(1+\alpha)}{\pi^{\alpha}}\left[-\frac{1}{k^{\alpha}} \frac{x^{2 \alpha}}{\Gamma(1+2 \alpha)} \cos _{\alpha}\left(k^{\alpha} x^{\alpha}\right)+\frac{1}{k^{2 \alpha}}\right. \\
& \times\left\{\frac{x^{\alpha}}{\Gamma(1+\alpha)} \sin _{\alpha}\left(k^{\alpha} x^{\alpha}\right)\right. \\
= & \left.-\frac{1}{\Gamma(1+2 \alpha)}\left(\frac{1}{k^{\alpha}}\left[\cos _{\alpha}\left(k^{\alpha} x^{\alpha}\right)-1\right]\right\}\right]\left.\right|_{0} ^{2 \pi} .
\end{aligned}
$$

Using (5) and (11), we give

$$
\begin{gathered}
b_{k}=\frac{\Gamma(1+\alpha)}{\pi^{\alpha}}{ }_{0} I_{2 \pi}^{(\alpha)} f(x) \cos _{\alpha}\left(k^{\alpha} x^{\alpha}\right) \\
=\frac{\Gamma(1+\alpha)}{\pi^{\alpha}}{ }_{0} I_{2 \pi}^{(\alpha)} \frac{t^{2 \alpha}}{\Gamma(1+2 \alpha)} \cos _{\alpha}\left(k^{\alpha} x^{\alpha}\right) \\
=\frac{\Gamma(1+\alpha)}{\pi^{\alpha}}\left[\frac{1}{k^{\alpha}} \frac{x^{2 \alpha}}{\Gamma(1+2 \alpha)} \sin _{\alpha}\left(k^{\alpha} x^{\alpha}\right)+\frac{1}{k^{2 \alpha}}\right. \\
\times\left[\frac{x^{\alpha}}{\Gamma(1+\alpha)} \cos _{\alpha}\left(k^{\alpha} x^{\alpha}\right)\right. \\
\left.\left.-\frac{1}{k^{\alpha}} \sin _{\alpha}\left(k^{\alpha} x^{\alpha}\right)\right]\right]\left.\right|_{0} ^{2 \pi}
\end{gathered}
$$


Therefore, $f(t)$ is expressed as follows:

$$
\begin{aligned}
f(x)= & \frac{\Gamma(1+\alpha)}{\Gamma(1+3 \alpha)}(2 \pi)^{2 \alpha} \\
& -\frac{\Gamma(1+\alpha)}{\Gamma(1+2 \alpha)} \sum_{k=1}^{\infty}\left(\frac{4 \pi}{k}\right)^{\alpha} \sin _{\alpha}\left(k^{\alpha} x^{\alpha}\right) \\
& +\sum_{i=1}^{\infty}\left(\frac{2}{k^{2}}\right)^{\alpha} \cos _{\alpha}\left(k^{\alpha} x^{\alpha}\right) .
\end{aligned}
$$

Example 3. The nondifferentiable data defined on the Cantor sets in the following form is given by the local fractional Fourier series as follows:

$$
f(t)=\frac{t^{3 \alpha}}{\Gamma(1+3 \alpha)}, \quad(0 \leq t \leq 2 \pi)
$$

where the fractal dimension $\alpha$ is equal to $\ln 2 / \ln 3$.

Making use of (3) and (16), the local fractional Fourier series of $f(t)$ reads as follows:

$$
\begin{aligned}
a_{0} & =\frac{\Gamma(1+\alpha)}{(2 \pi)^{\alpha}}{ }_{0} I_{2 \pi}^{(\alpha)} f(x)=\frac{\Gamma(1+\alpha)}{(2 \pi)^{\alpha}}{ }_{0} I_{2 \pi}^{(\alpha)} \frac{t^{3 \alpha}}{\Gamma(1+3 \alpha)} \\
& =\frac{\Gamma(1+\alpha)}{\Gamma(1+4 \alpha)}(2 \pi)^{3 \alpha} .
\end{aligned}
$$

By applying (4) and (12), we get

$$
\begin{aligned}
a_{k}= & \frac{\Gamma(1+\alpha)}{\pi^{\alpha}}{ }_{0} I_{2 \pi}^{(\alpha)} f(x) \sin _{\alpha}\left(k^{\alpha} x^{\alpha}\right) \\
= & \frac{\Gamma(1+\alpha)}{\pi^{\alpha}}{ }_{0} I_{2 \pi}^{(\alpha)} \frac{t^{3 \alpha}}{\Gamma(1+3 \alpha)} \sin _{\alpha}\left(k^{\alpha} x^{\alpha}\right) \\
= & \frac{\Gamma(1+\alpha)}{\pi^{\alpha}} \\
& \times\left[-\frac{1}{k^{\alpha}} \frac{x^{3 \alpha}}{\Gamma(1+2 \alpha)} \cos _{\alpha}\left(k^{\alpha} x^{\alpha}\right)\right. \\
& +\frac{1}{k^{2 \alpha}} \frac{x^{2 \alpha}}{\Gamma(1+2 \alpha)} \sin _{\alpha}\left(k^{\alpha} x^{\alpha}\right) \\
& +\frac{1}{k^{3 \alpha}}\left(\frac{x^{\alpha}}{\Gamma(1+\alpha)} \cos _{\alpha}\left(k^{\alpha} x^{\alpha}\right)\right. \\
= & \frac{1}{\pi^{\alpha}}\left(\left(\frac{2 \pi}{k^{3}}\right)^{\alpha}-\frac{\Gamma(1+\alpha)}{\Gamma(1+2 \alpha)}\left(\frac{(2 \pi)^{3}}{k}\right)^{\alpha}\right) .
\end{aligned}
$$

By using (5) and (13), we have

$$
\begin{aligned}
& b_{k}= \frac{\Gamma(1+\alpha)}{\pi^{\alpha}}{ }_{0} I_{2 \pi}^{(\alpha)} f(x) \cos _{\alpha}\left(k^{\alpha} x^{\alpha}\right) \\
&= \frac{\Gamma(1+\alpha)}{\pi^{\alpha}}{ }_{0} I_{2 \pi}^{(\alpha)} \frac{t^{3 \alpha}}{\Gamma(1+3 \alpha)} \cos _{\alpha}\left(k^{\alpha} x^{\alpha}\right) \\
&=\frac{\Gamma(1+\alpha)}{\pi^{\alpha}}\left[\frac{1}{k^{\alpha}} \frac{x^{3 \alpha}}{\Gamma(1+3 \alpha)} \sin _{\alpha}\left(k^{\alpha} x^{\alpha}\right)\right. \\
& \quad-\frac{1}{k^{2 \alpha}} \frac{x^{2 \alpha}}{\Gamma(1+2 \alpha)} \cos _{\alpha}\left(k^{\alpha} x^{\alpha}\right) \\
&+\frac{1}{k^{3 \alpha}}\left\{\frac{x^{\alpha}}{\Gamma(1+\alpha)} \sin _{\alpha}\left(k^{\alpha} x^{\alpha}\right)\right. \\
&\left.\left.=-\frac{\Gamma(1+\alpha)}{\Gamma(1+2 \alpha)}\left(\frac{4 \pi}{k^{2}}\right)^{\alpha} \cdot \frac{1}{k^{\alpha}}\left[\cos _{\alpha}\left(k^{\alpha} x^{\alpha}\right)-1\right]\right\}\right]\left.\right|_{0} ^{2 \pi}
\end{aligned}
$$

Hence, from (18)-(28), the nondifferentiable signal can be expressed as follows:

$$
\begin{aligned}
f(x)= & \frac{\Gamma(1+\alpha)}{\Gamma(1+4 \alpha)}(2 \pi)^{3 \alpha} \\
& +\sum_{k=1}^{\infty} \frac{1}{\pi^{\alpha}}\left(\left(\frac{2 \pi}{k^{3}}\right)^{\alpha}-\frac{\Gamma(1+\alpha)}{\Gamma(1+2 \alpha)}\left(\frac{(2 \pi)^{3}}{k}\right)^{\alpha}\right) \\
& \times \sin _{\alpha}\left(k^{\alpha} x^{\alpha}\right) \\
& -\frac{\Gamma(1+\alpha)}{\Gamma(1+2 \alpha)} \sum_{i=1}^{\infty}\left(\frac{4 \pi}{k^{2}}\right)^{\alpha} \cos _{\alpha}\left(k^{\alpha} x^{\alpha}\right) .
\end{aligned}
$$

Example 4. Let us consider the data defined on Cantor sets

$$
f(t)=\frac{t^{4 \alpha}}{\Gamma(1+4 \alpha)}, \quad(0 \leq t \leq 2 \pi)
$$

to be expressed in local fractional Fourier series.

According to (3) and (16), we have

$$
\begin{aligned}
a_{0} & =\frac{\Gamma(1+\alpha)}{(2 \pi)^{\alpha}}{ }_{0} I_{2 \pi}^{(\alpha)} f(x)=\frac{\Gamma(1+\alpha)}{(2 \pi)^{\alpha}}{ }_{0} I_{2 \pi}^{(\alpha)} \frac{t^{4 \alpha}}{\Gamma(1+4 \alpha)} \\
& =\frac{\Gamma(1+\alpha)}{\Gamma(1+5 \alpha)}(2 \pi)^{4 \alpha} .
\end{aligned}
$$

From (4) and (14), we arrive at the following local fractional Fourier series coefficient:

$$
\begin{aligned}
a_{k} & =\frac{\Gamma(1+\alpha)}{\pi^{\alpha}}{ }_{0} I_{2 \pi}^{(\alpha)} f(x) \sin _{\alpha}\left(k^{\alpha} x^{\alpha}\right) \\
& =\frac{\Gamma(1+\alpha)}{\pi^{\alpha}}{ }_{0} I_{2 \pi}^{(\alpha)} \frac{t^{4 \alpha}}{\Gamma(1+4 \alpha)} \sin _{\alpha}\left(k^{\alpha} x^{\alpha}\right)
\end{aligned}
$$




$$
\begin{aligned}
=\frac{\Gamma(1+\alpha)}{\pi^{\alpha}}[ & -\frac{1}{k^{\alpha}} \frac{x^{4 \alpha}}{\Gamma(1+4 \alpha)} \cos _{\alpha}\left(k^{\alpha} x^{\alpha}\right) \\
& +\frac{1}{k^{2 \alpha}} \frac{x^{3 \alpha}}{\Gamma(1+3 \alpha)} \sin _{\alpha}\left(k^{\alpha} x^{\alpha}\right) \\
& -\frac{1}{k^{3 \alpha}} \frac{x^{2 \alpha}}{\Gamma(1+2 \alpha)} \cos _{\alpha}\left(k^{\alpha} x^{\alpha}\right) \\
& +\frac{1}{k^{4 \alpha}}\left\{\frac{x^{\alpha}}{\Gamma(1+\alpha)} \sin _{\alpha}\left(k^{\alpha} x^{\alpha}\right)\right. \\
=-\frac{\Gamma(1+\alpha)}{\pi^{\alpha}} & \left(\frac{\left(\left((2 \pi)^{4} / k\right)\right)^{\alpha}}{\Gamma(1+4 \alpha)}+\frac{\left((2 \pi)^{2} / k^{3}\right)^{\alpha}}{\Gamma(1+2 \alpha)}\right) .
\end{aligned}
$$

Using (5) and (15), we obtain the following local fractional Fourier series coefficient:

$$
\begin{aligned}
b_{k}=\frac{\Gamma(1+\alpha)}{\pi^{\alpha}}{ }_{0} I_{2 \pi}^{(\alpha)} f(x) \cos _{\alpha}\left(k^{\alpha} x^{\alpha}\right) \\
=\frac{\Gamma(1+\alpha)}{\pi^{\alpha}}{ }_{0} I_{2 \pi}^{(\alpha)} \frac{t^{4 \alpha}}{\Gamma(1+4 \alpha)} \cos _{\alpha}\left(k^{\alpha} x^{\alpha}\right) \\
=\frac{\Gamma(1+\alpha)}{\pi^{\alpha}}\left[\frac{1}{k^{\alpha}} \frac{x^{4 \alpha}}{\Gamma(1+4 \alpha)} \sin _{\alpha}\left(k^{\alpha} x^{\alpha}\right)\right. \\
-\frac{1}{k^{2 \alpha}} \frac{x^{3 \alpha}}{\Gamma(1+3 \alpha)} \cos _{\alpha}\left(k^{\alpha} x^{\alpha}\right) \\
+\frac{1}{k^{3 \alpha}} \frac{x^{2 \alpha}}{\Gamma(1+2 \alpha)} \sin _{\alpha}\left(k^{\alpha} x^{\alpha}\right) \\
+\frac{1}{k^{4 \alpha}}\left[\frac{x^{\alpha}}{\Gamma(1+\alpha)} \cos _{\alpha}\left(k^{\alpha} x^{\alpha}\right)\right. \\
=\frac{\left.-\frac{1}{k^{\alpha}} \sin _{\alpha}\left(k^{\alpha} x^{\alpha}\right)\right]\left.\right|_{0} ^{2 \pi}}{\pi^{\alpha}}\left(\frac{\left(2 \pi / k^{4}\right)^{\alpha}}{\Gamma(1+\alpha)}-\frac{\left((2 \pi)^{3} / k^{2}\right)^{\alpha}}{\Gamma(1+3 \alpha)}\right) .
\end{aligned}
$$

Hence, we obtain the following local fractional Fourier coefficient:

$$
\begin{aligned}
f(x)= & \frac{\Gamma(1+\alpha)}{\Gamma(1+5 \alpha)}(2 \pi)^{4 \alpha} \\
& -\sum_{k=1}^{\infty} \frac{\Gamma(1+\alpha)}{\pi^{\alpha}}\left(\frac{\left(\left((2 \pi)^{4} / k\right)\right)^{\alpha}}{\Gamma(1+4 \alpha)}+\frac{\left((2 \pi)^{2} / k^{3}\right)^{\alpha}}{\Gamma(1+2 \alpha)}\right)
\end{aligned}
$$

$$
\begin{aligned}
& \times \sin _{\alpha}\left(k^{\alpha} x^{\alpha}\right) \\
& +\sum_{i=1}^{\infty} \frac{\Gamma(1+\alpha)}{\pi^{\alpha}}\left(\frac{\left(2 \pi / k^{4}\right)^{\alpha}}{\Gamma(1+\alpha)}-\frac{\left((2 \pi)^{3} / k^{2}\right)^{\alpha}}{\Gamma(1+3 \alpha)}\right) \\
& \times \cos _{\alpha}\left(k^{\alpha} x^{\alpha}\right) .
\end{aligned}
$$

\section{Conclusions}

Local fractional Fourier series are a generalization for Fourier series defined on the Cantor sets based upon the local fractional calculus. In this work, we use the local fractional Fourier series to deal with the nondifferentiable data defined on the Cantor sets. Some explicit computations for nondifferentiable data defined on the Cantor sets are also given to show the efficiency of the present method.

\section{Conflict of Interests}

The authors declare that there is no conflict of interests regarding the publication of this paper.

\section{Acknowledgment}

The work was supported by the National Science Fund for Distinguished Young Scholar (60025102).

\section{References}

[1] J. T. Machado, V. Kiryakova, and F. Mainardi, "Recent history of fractional calculus," Communications in Nonlinear Science and Numerical Simulation, vol. 16, no. 3, pp. 1140-1153, 2011.

[2] J. Tenreiro Machado, A. M. Galhano, and J. J. Trujillo, "Science metrics on fractional calculus development since 1966," Fractional Calculus and Applied Analysis, vol. 16, no. 2, pp. 479-500, 2013.

[3] R. Metzler, W. G. Glöckle, and T. F. Nonnenmacher, "Fractional model equation for anomalous diffusion," Physica A: Statistical Mechanics and Its Applications, vol. 211, no. 1, pp. 13-24, 1994.

[4] R. Metzler and J. Klafter, "The random walk's guide to anomalous diffusion: a fractional dynamics approach," Physics Report, vol. 339, no. 1, pp. 1-77, 2000.

[5] A. S. Kozlov, D. Andor-Ardó, and A. J. Hudspeth, "Anomalous Brownian motion discloses viscoelasticity in the ear's mechanoelectrical-transduction apparatus," Proceedings of the National Academy of Sciences, vol. 109, no. 8, pp. 2896-2901, 2012.

[6] E. C. de Oliveira, F. Mainardi, and J. Vaz Jr., "Models based on Mittag-Leffler functions for anomalous relaxation in dielectrics," The European Physical Journal Special Topics, vol. 193, no. 1, pp. 161-171, 2011.

[7] G. M. Zaslavsky, "Chaos, fractional kinetics, and anomalous transport," Physics Report, vol. 371, no. 6, pp. 461-580, 2002.

[8] M. Hellmann, D. W. Heermann, and M. Weiss, "Anomalous reaction kinetics and domain formation on crowded membranes," Europhysics Letters, vol. 94, no. 1, Article ID 18002, 2011.

[9] H. Sheng, Y. Q. Chen, and T. S. Qiu, Fractional Processes and Fractional-Order Signal Processing, Springer, London, UK, 2012. 
[10] S. Das and I. Pan, Fractional Order Signal Processing: Introductory Concepts and Applications, Springer, 2012.

[11] M. D. Ortigueira, Fractional Calculus for Scientists and Engineers, Springer, 2011.

[12] M. D. Ortigueira and J. A. Tenreiro Machado, "Fractional signal processing and applications," Signal Processing, vol. 83, no. 11, pp. 2285-2286, 2003.

[13] R. Magin, M. D. Ortigueira, I. Podlubny, and J. J. Trujillo, "On the fractional signals and systems," Signal Processing, vol. 91, no. 3, pp. 350-371, 2011.

[14] M. D. Ortigueira, J. A. Machado, J. J. Trujillo, and B. M. Vinagre, "Fractional signals and systems," Signal Processing, vol. 91, no. 3, p. 349, 2011.

[15] Y. Chen, R. Sun, A. Zhou, and N. Zaveri, "Fractional order signal processing of electrochemical noises," Journal of Vibration and Control, vol. 14, no. 9-10, pp. 1443-1456, 2008.

[16] R. Tao, B. Deng, and Y. Wang, "Research progress of the fractional Fourier transform in signal processing," Science in China F: Information Sciences, vol. 49, no. 1, pp. 1-25, 2006.

[17] M. F. Lima, J. T. Machado, and M. Crisóstomo, "Robotic manipulators with vibrations: short time Fourier transform of fractional spectra," in Intelligent Engineering Systems and Computational Cybernetics, pp. 49-60, Springer, Amsterdam, The Netherlands, 2009.

[18] E. Dinç and D. Baleanu, "Fractional wavelet transform for the quantitative spectral resolution of the composite signals of the active compounds in a two-component mixture," Computers and Mathematics with Applications, vol. 59, no. 5, pp. 1701-1708, 2010.

[19] R. R. Nigmatullin, J. T. Machado, and R. Menezes, "Self-similarity principle: the reduced description of randomness," Central European Journal of Physics, vol. 11, no. 6, pp. 724-739, 2013.

[20] R. R. Nigmatullin, S. I. Osokin, and V. A. Toboev, "NAFASS: discrete spectroscopy of random signals," Chaos, Solitons and Fractals, vol. 44, no. 4-5, pp. 226-240, 2011.

[21] D. Baleanu, R. Nigmatullin, O. Suleyman, and S. Cetin, "Application of the Prony's method to analysis of the FTIR data," Fractional Differentiation and Its Applications, vol. 6, no. 1, pp. 581-586, 2013.

[22] D. Baleanu and X. J. Yang, "Local fractional Fourier series with applications to representations of fractal signals," in Proceedings of the ASME/IEEE MESA Conference, Portland, Ore, USA, 2013.

[23] X. J. Yang, D. Baleanu, and J. T. Machado, "Application of the local fractional Fourier series to fractal signals," in Proceedings of the Discontinuity and Complexity in Nonlinear Physical Systems, J. A. Tenreiro Machado, D. Baleanu, and A. C. J. Luo, Eds., Springer, 2014.

[24] Y.-J. Yang, D. Baleanu, and X.-J. Yang, "Analysis of fractal wave equations by local fractional Fourier series method," Advances in Mathematical Physics, vol. 2013, Article ID 632309, 6 pages, 2013.

[25] Y. Zhao, D. Baleanu, M. Baleanu, D. F. Cheng, and X. J. Yang, "Mappings for special functions on Cantor sets and special integral transforms via local fractional operators," Abstract and Applied Analysis, vol. 2013, Article ID 316978, 6 pages, 2013.

[26] M.-S. Hu, R. P. Agarwal, and X.-J. Yang, "Local fractional Fourier series with application to wave equation in fractal vibrating string," Abstract and Applied Analysis, vol. 2012, Article ID 567401, 15 pages, 2012.

[27] Y. Zhang, A. Yang, and X. J. Yang, "1-D heat conduction in a fractal medium A solution by the local fractional Fourier series method," Thermal Science, vol. 17, no. 3, pp. 953-956, 2013.
[28] X.-J. Yang, D. Baleanu, H. M. Srivastava, and J. A. Tenreiro Machado, "On local fractional continuous wavelet transform," Abstract and Applied Analysis, vol. 2013, Article ID 725416, 5 pages, 2013.

[29] Y. Zhao, D. Baleanu, C. Cattani, D.-F. Cheng, and X.-J. Yang, "Local fractional discrete wavelet transform for solving signals on Cantor sets," Mathematical Problems in Engineering, vol. 2013, Article ID 560932, 6 pages, 2013.

[30] X. J. Yang, Advanced Local Fractional Calculus and Its Applications, World Science, New York, NY, USA, 2012. 


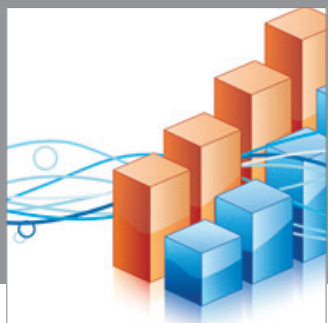

Advances in

Operations Research

mansans

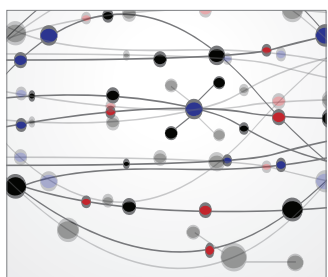

The Scientific World Journal
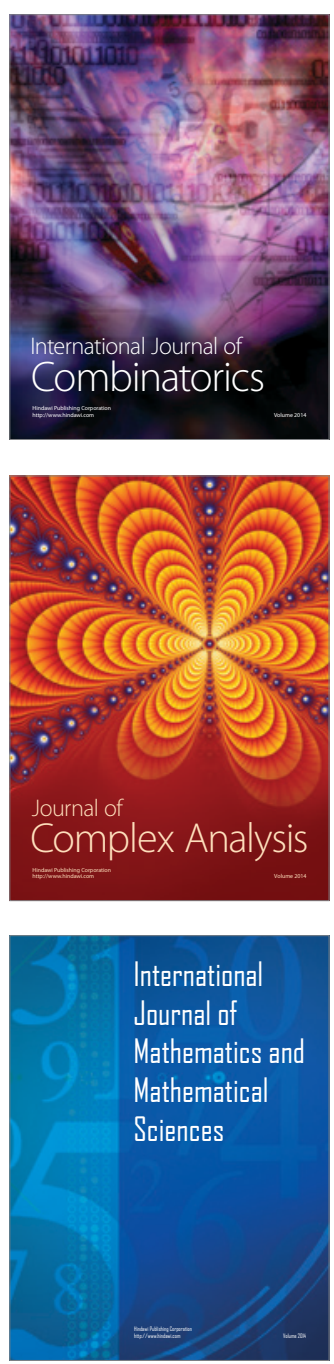
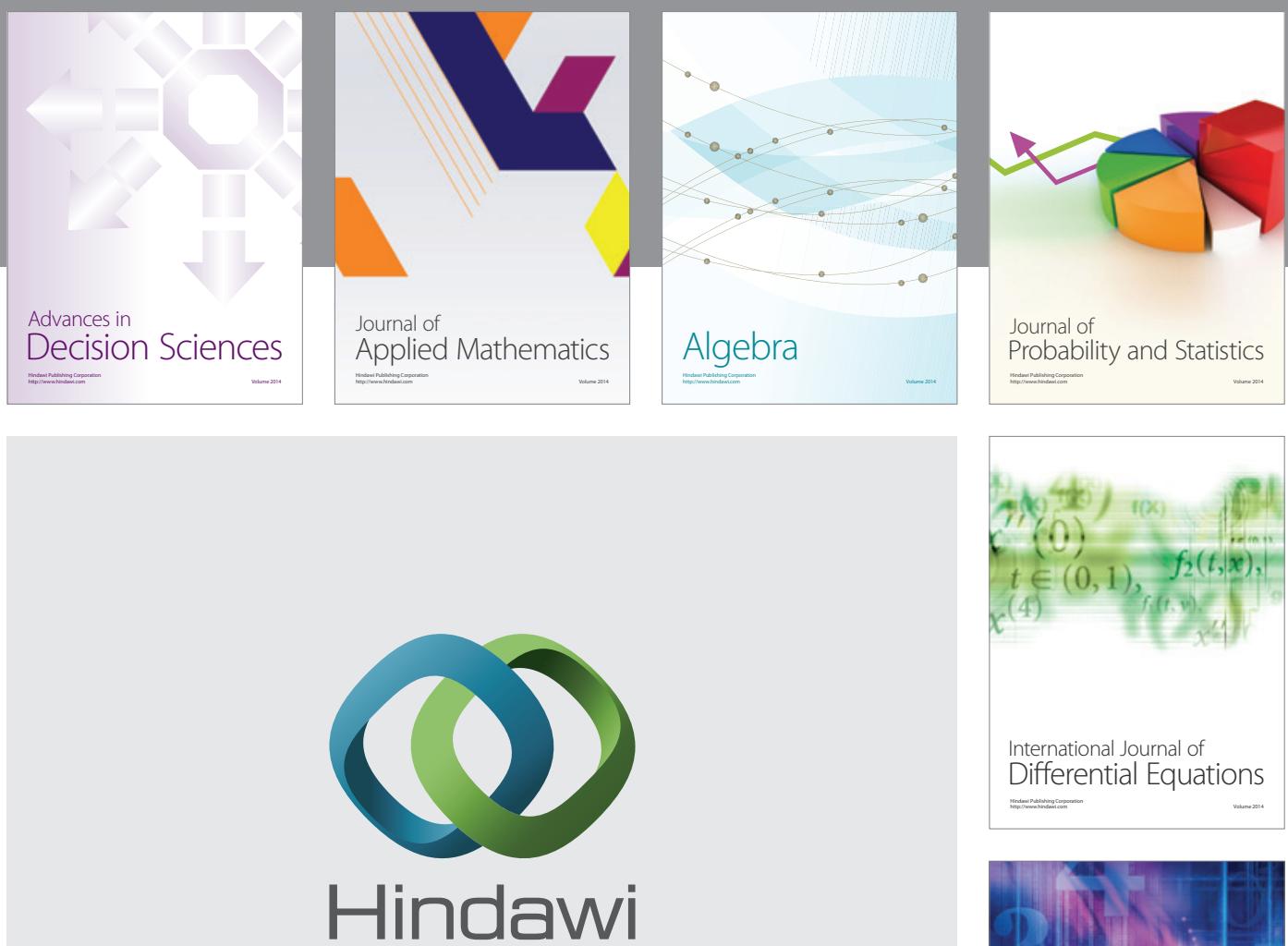

Submit your manuscripts at http://www.hindawi.com
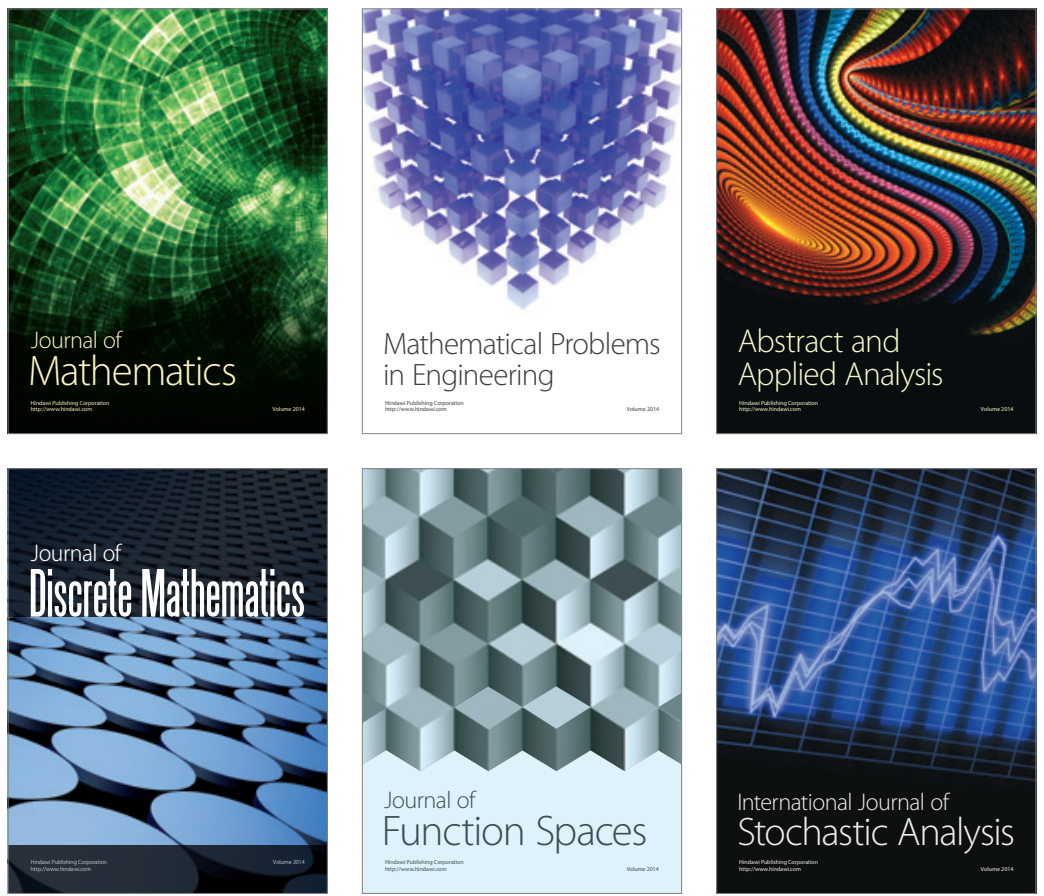

Journal of

Function Spaces

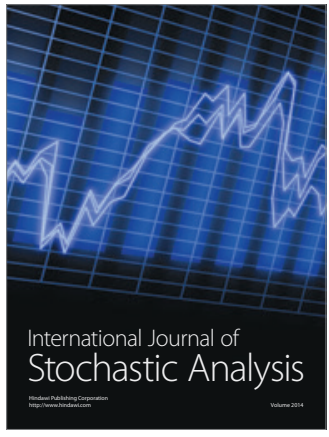

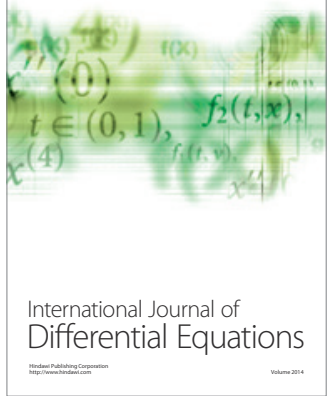
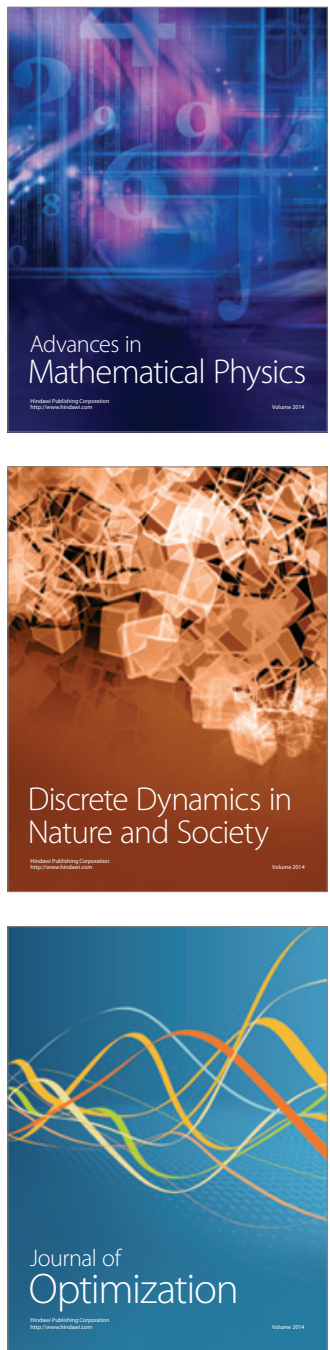